\title{
FUNDAMENTALISMO RELIGIOSO ENTRE JÓVENES UNIVERSITARIOS DE COSTA RICA: CONSERVADURISMO POLÍTICO Y ESPIRITUALIDAD SIN RELIGIÓN $N^{1}$
}

\section{RELIGIOUS FUNDAMENTALISM AMONG COSTA RICAN UNIVERSITY STUDENTS: POLITICAL CONSERVATISM AND SPIRITUALITY WITHOUT RELIGION}

Javier Tapia Valladares*

Mijail Rojas Carvajal**

Melissa Villalobos García***

RESUMEN

El presente artículo constituye una aproximación psicosocial a la cuestión del fundamentalismo religioso, en una muestra de jóvenes universitarios. Se analizan el autoritarismo, la confianza en las instituciones, el afecto y la satisfacción vital, la afiliación y las prácticas religiosas, el nivel de apertura a la búsqueda existencial y otras dimensiones. Estas son examinadas en su grado de predicción del fundamentalismo religioso a partir de análisis estadísticos, los cuales permiten extraer conclusiones sobre configuraciones psicosociales, algunas de las cuales se organizan en torno al género.

PALABRAS CLAVE: COSTA RICA * RELIGIÓN * FUNDAMENTALISMO RELIGIOSO * ESTUDIANTE UNIVERSITARIO * JOVEN * PSICOLOGÍA DE LA RELIGIÓN

1 Esta investigación recibió el apoyo del Instituto de Investigaciones Psicológicas (IIP) y de la Vicerrectoría de Investigación de la Universidad de Costa Rica (UCR), por medio de los fondos asignados en la actividad de investigación 723-B0-240, a cargo del Prof. Javier Tapia, PhD.

* Instituto de Investigaciones Psicológicas (IIP), Escuela de Psicología de la Universidad de Costa Rica (UCR). javier.tapiav@gmail.com

** Área de Neurobiología, Centro de Investigación en Neurociencias (CIN) de la Universidad de Costa Rica (UCR). mijail.rojas.ca@gmail.com / mijail.rojas@ucr.ac.cr

*** Fundación Omar Dengo, Costa Rica. meliv09@gmail.com 


\section{ABSTRACT}

This article is a psychosocial approach to religious fundamentalism among university students. We analyze the authoritarianism, confidence in political institutions, affect and satisfaction with life, religious affiliation and practices, level of existential quest and others variables. These dimensions are analyzed in its prediction level of religious fundamentalism with statistical analyses. It's concluded about psychosocial configurations, some organized around gender.

\section{KEYWORDS: COSTA RICA * RELIGION * RELIGIOUS FUNDAMENTALISM * UNIVERSITY STUDENT * YOUTH * PSYCHOLOGY OF RELIGION}

\section{INTRODUCCIÓN ${ }^{2}$}

El presente artículo busca dar cuenta de algunas de las preguntas de investigación planteadas para el capítulo de Costa Rica, en el marco del proyecto de investigación internacional sobre fundamentalismo religioso ${ }^{3}$. En esta etapa de esta investigación internacional conjunta, solo es posible hacer referencia a las

$2 \quad$ El primer autor de este artículo desea agradecer el trabajo de preparación y traducción de los instrumentos por parte de los psicólogos Lic. Ricardo Castro y Br. Melissa Villalobos, así como al sociólogo Lic. Danilo Reuben. Además, agradezco a los estudiantes de psicología quienes participaron en la recolección de los datos, a saber: Mijail Rojas, Edison Chavarría, Valeria Sancho y Nelson Jiménez. La coordinación excelente del trabajo de campo, así como, la sistematización de las bases de datos fue realizada por la psicóloga Br. Melissa Villalobos a quien manifiesto mi gratitud y reconocimiento. También cabe nuestra inmensa gratitud al Dr. Mauricio Molina, colega de nuestro Instituto, quién nos ofreció una importante asesoría estadística para la última versión del artículo. configuraciones nacionales, relacionadas con las variables psicosociales y psicoreligiosas vinculadas al fundamentalismo religioso y será posteriormente, en otros trabajos futuros, en los cuales se podrán comparar los hallazgos en otros países, con los datos costarricenses.

El fundamentalismo religioso se ha investigado ampliamente en el ámbito internacional, lo cual sirve de contexto a esta investigación internacional concertada (Altemeyer y Hunsberger, 1992). Esto contrasta con la investigación en ciencias sociales sobre el fundamentalismo religioso cristiano en Costa Rica, la cual ha sido escasa o nula. En la Sociología, el trabajo de Solís (2008) es el más reciente, aún cuando no trata el tema directamente, mientras que en la Antropología, los trabajos conocidos (Schäfer, 1992) corren el riesgo de haber perdido vigencia o tratan el tema solo de forma tangencial.

En el campo de las investigaciones en Psicología, acerca del Fundamentalismo Religioso (FR), encontramos el trabajo de Rodríguez (2007), en el cual se estudia si el fundamentalismo religioso se conforma junto a otros aspectos, como predictor de las actitudes hacia la Nación. En dicho trabajo, con jóvenes adolescentes escolarizados de una zona urbana, se logra encontrar una relación tenue entre el fundamentalismo y la identidad nacional y el nacionalismo, pero no se encuentra relación alguna con el patriotismo. Cuando Rodríguez (2007) profundiza su análisis de las relaciones del fundamentalismo nos muestra, en definitiva, que el fundamentalismo no logra explicar aspectos particulares ni de la identidad nacional ni del nacionalismo. Sus hallazgos evidencian como en la muestra de jóvenes escolarizados el fundamentalismo 
cristiano posee un rol poco o nada significativo, al intentar explicar las actitudes psicosociales de identidad nacional y nacionalismo. Cabe preguntarse si la limitación metodológica del tipo de análisis del fundamentalismo podría incidir en los resultados obtenidos por Rodríguez (2007), de ahí que en este estudio, recurriendo a una mayor variedad en la aproximación metodológica, podríamos lograr obtener una descripción más amplia del fundamentalismo religioso.

Ahora bien, ¿a qué se refiere la psicología de la religión cuando trata la cuestión del fundamentalismo religioso? Es cierto que el trabajo de Hood, Hill y Williamson (2005; Watson, Chen y Hood, 2011) ha representado un avance en la comprensión del fundamentalismo, pero hasta donde sabemos, solo en términos conceptuales. Para los fines de esta investigación, sin duda el trabajo de Altemeyer y Hunsberger (2003) resulta provisionalmente más pertinente, en particular por definir mejor el campo y alcance de la investigación en la psicología del fundamentalismo religioso en un sentido empírico.

Altemeyer y Hunsberger $(1992,2003)$ han establecido que el fundamentalismo religioso es:

... la creencia que existe un conjunto de enseñanzas las cuales contienen claramente la verdad fundamental, básica, intrínseca, esencial, fija, acerca de lo humano y lo divino; una verdad esencial que estaría fundamentalmente opuesta a las fuerzas del mal contra las cuales hay que luchar; una verdad que debe ser seguida en la actualidad, conforme a las prácticas fundamentales del pasado $y$ que quienes creen $y$ siguen estas enseñanzas fundamentales, tienen una relación especial con Dios (1992: 118).

Es decir, que el fundamentalismo religioso parece reflejar un sistema cerrado, dogmático y aparentemente prejuiciado respecto a otros grupos sociales.

Sin embargo, a pesar de esta base descriptiva, no existe un consenso sobre dicho concepto, ya que diversos autores mencionan características que le son transversales (Altemeyer y Hunsberg, 1992; Altemeyer, 2003; Galen, Wolfe, Deleeuw y Wyngarden, 2009;
Jakubowska y Oniszczenko, 2010; Saroglou y Muñoz-García, 2008). De ahí es posible añadir, a partir de varias definiciones y características propuestas en esta literatura, que el fundamentalismo religioso puede ser conceptuado como una dimensión de la religiosidad, como sistema centralizado de significados, el cual enfatiza la forma de mantener o persistir en creencias religiosas inmodificables.

Así, la forma de las creencias es independiente de su contenido, emergiendo el fundamentalismo como meta-creencia. Las meta-creencias poseen características cognoscitivas mediadoras de procesos de información convergente, pero también son de baja complejidad de pensamiento en temas relacionados con argumentos existenciales (Van Pachterbeke, Keller y Saroglou, en prensa).

Desde el punto de vista de los hallazgos empíricos, sabemos que el fundamentalismo religioso tiende a exacerbar el sesgo confirmatorio de la creencia religiosa particular, mostrando baja apertura a nuevas experiencias y altos niveles de autoritarismo (Saroglou y MuñozGarcía, 2008). También se ha encontrado la elevada internalización de la religiosidad, rangos elevados de etnocentrismo y correlatos positivos con los prejuicios (Núñez-Alarcón, MorenoJiménez y Moral-Toranzo, 2011), así como discriminación hacia otros (Altemeyer y Hunsberg, 1992; Altemeyer, 2003; Jakubowska y Oniszczenko, 2010). Además, brinda el fundamentalismo religioso un esquema o andamiaje sobre el cual se instaura la creencia, ofreciendo una forma de interpretación al contenido específico de esta. Así, la meta-creencia presenta una elaboración del modelo de interpretación a partir de la creencia misma, clasificando y organizando las representaciones e interpretaciones de los eventos (Galen, Wolfe, Deleeuw y Wyngarden, 2009; Gribbins y Vandenberg, 2011).

Respecto a la relación con los otros, en el fundamentalismo la discriminación y el prejuicio contra el exogrupo, es elaborada en función del contenido del esquema más allá del esquema mismo. Estos patrones tienen sentido a partir del contenido de las creencias en sí, basadas en un tipo de derogación de los otros explícita o implícita (Kirkpatric, 2005). Sin 
embargo, hallazgos experimentales matizan la explicación de Kirkpatric. Por ejemplo, Blogowska y Saroglou (2011) encuentran evidencia de niveles de prosocialidad hacia el exogrupo cuando este no es un objetivo cercano a los individuos con creencia fundamentalista, lo cual abre algunas paradojas y matices.

Aún cuando Jakubowska y Oniszczenko (2010) describen que podrían existir factores genéticos implicados en las actitudes hacia la religiosidad, se acepta que las características del fundamentalismo son trasmitidas mediante aprendizaje social. Kinnval (2004) menciona que el acoger dogmas podría referirse a prácticas o formas de afrontar la incertidumbre, la ansiedad $y$ la amenaza.

Sin embargo, los procesos de aprendizaje social son diferenciados según el contexto. En Costa Rica, una importante proporción de la juventud continúa teniendo contacto con las creencias y las prácticas religiosas, ya sea porque esta forma parte de diferentes componentes de la tradición cultural o porque las personas jóvenes participan directamente en el ámbito religioso. De hecho, sabemos que de una muestra nacional costarricense, en el rango de los 15 a los 17 años, el 30,60\% de los jóvenes de zona urbana y el 27,30\% de los jóvenes de zona rural, participan en grupos religiosos. Lo mismo ocurre con los jóvenes en el rango de edad de los 18 a los 24 años, a saber, el 22,70\% de la zona urbana y el 18,60\% de la zona rural participan en grupos religiosos. En ambos segmentos de edad, este nivel de participación es el segundo en importancia después de la participación en grupos deportivos (Consejo Nacional de Política Pública de la Persona Joven, 2008: 91). Por otro lado, es importante subrayar que el 43,90\% de las jóvenes y el 31,70\% de los jóvenes afirmaron que los hijos y la familia es el motivo más importante al dar sentido a su vida, mientras para el 33,50\% de las jóvenes y el 31\% de los jóvenes, Dios aparece como el segundo aspecto que le da sentido a su vida (p. 85). Estos datos nos señalan la menor importancia de la participación en actividades socio-políticas y de activismo social de la juventud costarricense; asimismo, destacan la importancia para los jóvenes de los valores como la familia y las creencias religiosas al definir lo que da sentido a su vida.

En este estudio, se podrá observar las semejanzas de la juventud costarricense en general, con respecto a las características presentes en una muestra de jóvenes universitarios. En efecto, habría dos preguntas pertinentes a la realidad de la juventud urbana, escolarizada y de clase media, del Valle Central de Costa Rica, que asiste a la principal universidad pública del país. Por una parte, ¿cuáles son las características psicosociales y psicoreligiosas de una muestra de jóvenes universitarios? Por otra parte, ¿cómo se configura el fundamentalismo religioso con algunas dimensiones relevantes de tipo socio-cognoscitivas y psicoreligiosas entre jóvenes universitarios?

Para examinar estas preguntas de investigación vamos a seguir tres objetivos. En primer lugar, se van a describir algunas de las características psicosociales de estos jóvenes universitarios relacionadas con el autoritarismo, la confianza en las instituciones, el afecto y la satisfacción vital. En segundo lugar, se trazará una suerte de perfil psicoreligioso de la muestra estudiada, al evidenciar su afiliación y prácticas religiosas, su nivel de apertura a la búsqueda existencial y su posicionamiento frente al fundamentalismo religioso. Aún cuando estos aspectos psicoreligiosos y psicosociales no agotan lo que es posible examinar, sí abordan algunos de las cuestiones menos estudiadas en la investigación internacional. En tercer lugar, se examinarán las asociaciones y el grado de predicción que poseen respecto al fundamentalismo religioso, las dimensiones del autoritarismo, la confianza en las instituciones, el afecto, el nivel de satisfacción con la vida, así como, cuatro dimensiones fundamentales de la religiosidad.

\section{METODOLOGÍA}

\section{PARTICIPANTES}

En este estudio participaron 184 estudiantes de pregrado pertenecientes tanto a la Facultad de Ingeniería como a la Facultad de Ciencias Sociales de la Universidad de Costa Rica. Esta muestra fue escogida de manera 
deliberada, según los criterios establecidos con el grupo internacional. Su edad promedio es de 20,53 años, siendo la media para los varones $(\mathrm{n}=117 ; 66,5 \%)$ de 20,82 y para las mujeres $(\mathrm{n}=59 ; 33,5 \%)$ de 19,97 años. Al preguntar a las personas participantes cuál era su mayor grado académico o su campo actual de estudio, respondieron: Ingeniería ( $\mathrm{n}=70 ; 35 \%)$, Ciencias Sociales $(n=68 ; 34 \%)$, Derecho $(n=14 ; 7 \%)$, Arte $(\mathrm{n}=5 ; 2,55 \%)$, Ciencias Naturales $(\mathrm{n}=5 ; 2,5 \%)$, Ciencias Económicas ( $\mathrm{n}=2 ; 1,0 \%)$, Humanidades $(n=1 ; 5 \%)$, Ciencias Religiosas $(n=1 ; 0,5 \%)$. Sin embargo, para simplificar algunos análisis vamos a considerar dos grandes áreas: por un lado, la de Ingeniería y Ciencias Naturales $(n=75 ; 40,8 \%)$ y por otro, la de Ciencias Sociales y Humanas ( $n=91 ; 49,5 \%)$, en la cual se incluyen los individuos que eligieron Arte, Derecho, Ciencias Económicas, Humanidades y Ciencias Religiosas.

Entre esta muestra de jóvenes universitarios se encuentran 65 personas católicas (41,9\%), 19 evangélicas (12,3\%), 29 agnósticas $(18,7 \%)$ y 12 ateas (7,7\%). Es importante señalar que durante la aplicación del cuestionario no se ofreció ninguna aclaración acerca de la posible confusión entre el significado del agnosticismo y el del ateísmo; las personas respondieron a esto según su propio juicio. En la categoría Otra, se encontró a 30 personas $(19,4 \%)$ y en la de No sabe o no responde a 29 (14,5\%). Ahora bien, si estos resultados se evalúan en términos de dos categorías, la de creyentes y la de no creyentes, tendríamos que 84 personas $(54,2 \%)$ serían creyentes, mientras que 41 personas $(26,4 \%)$ serían no creyentes. Aún cuando las personas que no saben responder junto con las personas en la categoría Otra afiliación alcanza casi el 34\%, no es posible determinar el significado de estos dos últimos datos o si el peso que representan podría atribuirse a la categoría de Creyentes o de No creyentes. De estos datos se extrajo que aún cuando parece existir una disminución en la cantidad de personas creyentes $y$ un aumento de las personas no creyentes, en realidad parece mantenerse la tendencia de otros datos nacionales (Consejo Nacional de la Política Pública de la Persona Joven, 2008), la cual sigue mostrando una cierta mayoría de afiliación al cristianismo en Costa Rica. Sin embargo, en esta muestra de estudiantes universitarios, esta tendencia podría estar matizada por esas 59 personas ubicadas en la categoría de Otra y en la de No sabe o no responde, lo cual es indiscernible dentro de los límites de este estudio, pues se desconoce hacia dónde se orientaría dicha tendencia.

\section{INSTRUMENTOS}

En este estudio se han aplicado doce instrumentos diferentes. Sin embargo, para este artículo se ha trabajado solo con ocho de ellos, los cuales se consideran pertinentes para la pregunta de investigación que nos ocupa. Así, pues se presentan dos tipos de instrumentos, tanto para evaluar dimensiones psicosociales relacionadas con el fundamentalismo religioso, como para evaluar su relación con aspectos psicoreligiosos. A continuación se hace una descripción de estos instrumentos psicométricos.

$\diamond \quad$ Escala de Autoritarismo de Derecha (Right-Wing Authoritarianism Scale -RWAS. Funke, 2005). Se trata de una escala tipo Likert con opciones de respuesta desde 1 (totalmente en desacuerdo) hasta 7 (totalmente de acuerdo). Esta posee como objetivo evaluar el autoritarismo de derecha, al conocer el nivel de agresividad (4 ítems), sumisión (4 ítems) y conservadurismo (4 ítems) de la persona que responde la escala. En esta investigación, hemos obtenido los siguientes índices de consistencia interna para este instrumento: agresividad: 0,58 (eliminando ítem 4), sumisión: 0,57 (eliminando ítem 8) y conservadurismo: 0,59.

Dado que encontramos índices de consistencia interna con puntaje deficiente, se realizó un análisis factorial con rotación Varimax a la escala. Se obtienen cuatro factores con autovalores superiores a 1 , los cuales agrupan de forma disímil a los elementos del factor según las expectativas de la escala original. El primer factor agrupa a los ítems 1, 2, 5, 6, 9 y 10, con una explicación de la varianza en la matriz 
rotada del 25\%. El segundo factor agrupa los ítems 4, 11 y 12, con una explicación de la varianza de la matriz rotada de $16 \%$. El tercer factor agrupa a los ítems 7 y 8 , con una explicación de la varianza de la matriz rotada del 11\%. El cuarto y último factor agrupa al ítem número 3, con una explicación de la varianza de la matriz rota de $11 \%$, para una explicación de la varianza acumulada del $63 \%$.

Esto implicó la realización de un segundo análisis factorial a partir de los indicadores brindados por la matriz de correlaciones y el gráfico de sedimentación de auto-valores del primer análisis factorial. En este último, la curva de sedimentación se estabiliza y detiene su caída posterior al factor 2 , lo cual indica una debilidad en la conformación de los factores $3 y$ 4 (Mayers, Gamst y Guarino, 2006).

En el segundo análisis factorial con rotación Varimax, se determina el número de factores a determinar en 2 . El resultado muestra un primer factor que agrupa a los ítems 1, 2, 3, 5, 6 y 10, con una varianza explicada en la matriz rotada de $24 \%$, mientras que en el segundo factor se agrupan los ítems 4, 7, 8, 9, 11 y 12, con una explicación de la varianza en la matriz rotada de $19 \%$, para una varianza total del $43 \%$. Cabe destacar que el índice de consistencia interna para el primer factor es de 0,75 , mientras que el segundo factor obtuvo una puntuación de 0,64 .

Si bien, la conformación de estos dos factores se deriva de forma satisfactoria, no se cuentan con criterios teóricos ni de estandarización de la escala para tomarlos como tales, por lo que no se utilizan estos dos factores como elementos independientes. En su lugar, se realiza un tercer análisis factorial con rotación Varimax, del que se extrae solamente un factor, el cual explica un $28 \%$ de la varianza. Esta obtiene índice de consistencia interna de 0,76 para la escala completa.

Tal como lo describen Tabachnick y Fidell (2006), se toma la solución que aporte un mejor ajuste, pero que a la vez, no atente contra la parsimonia. Por tanto y como se ha descrito, la falta de evidencia empírica y teórica que respalde el establecimiento de dos factores o cuatro factores nos ha conducido a tomar una postura más conservadora. Por tanto, los análisis posteriores se realizarán a partir de las subescalas originales descritas anteriormente. A pesar de esto, se brindará un análisis alternativo a partir de la escala completa del RWAS descrita en el último análisis factorial.

$\diamond \quad$ Escala de Orientación e Intereses Políticos (Political Interest and Orientation. Halman, 2001). Se trata de una escala Likert, la cual intenta determinar el nivel de confianza de las personas en instituciones como: la Asamblea Legislativa, la policía, los políticos, los partidos políticos y la Organización de las Naciones Unidas. La escala de respuestas va desde 0 (no confío para nada) hasta 10 (confío totalmente). Para esta escala en esta investigación se ha obtenido un índice de consistencia interna de 0,84 . Muy relacionada con esta escala y colocada inmediatamente después, se incluyó una pregunta en la cual la persona tenía que ubicarse en una escala de 0 a 10 , en la que 0 significa izquierda política y 10 derecha política.

$\diamond \quad$ Escala de Afecto Positivo y Negativo (The International Positive and Negative Affect Schedule Short Form -I-PANASSF. Thompson, 2007). Con esta escala se establece el estado de ánimo predominante de la persona que responde. Cuenta con 5 ítems para la subescala de afecto positivo y otros 5 ítems para la subescala de afecto negativo. Se presentan 5 emociones por cada subescala $y$ se responde cada ítem desde 1 (nunca) hasta 5 (siempre). En el presente estudio, se ha obtenido un índice de consistencia interna alpha de Cronbach de 0,74 para el afecto positivo $y$ de 0,75 para el afecto negativo.

$\diamond \quad$ Escala de Satisfacción con la Vida (The Satisfaction with Life Scale. Diener, Emmons, Larsen y Griffin, 1985). Esta escala posee 5 ítems y establece el nivel de satisfacción con la vida de la persona. Las 
opciones de respuesta van desde 1(totalmente en desacuerdo) hasta 7 (totalmente de acuerdo). En el presente estudio, se ha obtenido un índice de consistencia interna alpha de Cronbach de 0,87 para esta escala.

Afiliación Religiosa. Para obtener los datos más básicos sobre la afiliación religiosa, se incluyó una sola pregunta, en la cual se indaga la afiliación o convicción religiosa actual de la persona. Existían las siguientes posibilidades de elección: católico(a), evangélico(a), judío(a), musulmán(a), budista, agnóstico(a), ateo(a), católico(a) no practicante, otra (la cual tenían que escribir según su preferencia).

Escala de Prácticas Religiosas (Religiosity Scale. Saroglou y Muñoz-García, 2008). Con 3 ítems en una escala de tipo Likert de 1 (totalmente en desacuerdo) a 7 (totalmente de acuerdo), sobre la importancia de la religión, Dios y la espiritualidad para la persona, establece qué tan importante son esos aspectos para cada una de ellas. Se ha obtenido un índice de consistencia interna de 0,82 . También se relaciona con esta escala una pregunta añadida pero diferente, sobre la frecuencia de la práctica de rezar u orar desde 1 (nunca) hasta 7 (todos los días).

$\diamond \quad$ Escala de Cuatro Polos de la Religiosidad (Four Poles Religiosity Scale. Saroglou, 2011). Esta escala busca captar el grado de religiosidad de la persona en cuatro dimensiones fundamentales. Posee 12 ítems, 3 para cada una de las siguientes subescalas: creencias $(\alpha=0,84)$, rituales y emociones manifiestas $(\alpha=0,88)$, reglas morales $(\alpha=0,87)$, comunidad $y$ sentido de agrupación $(\alpha=0,85)$. También las opciones de respuesta van desde 1 (totalmente en desacuerdo) hasta 7 (totalmente de acuerdo).
Escala de Fundamentalismo Religioso (Religious Fundamentalism Scale.
Altemeyer y Hunsberger, 2004). Este instrumento ofrece una visión de la posición de la persona acerca de algunas ideas asociadas al fundamentalismo religioso. Se trata de 12 ítems los cuales se responden en una escala de 1 (totalmente en desacuerdo) a 7 (totalmente de acuerdo). En este caso se ha obtenido para la actual investigación un índice de consistencia interna de 0,86.

$\diamond \quad$ Escala de Búsqueda Existencial (Existential Quest Scale. Van Pachterbeke, Keller y Saroglou, en prensa). Tiene 9 ítems los cuales se responden en una escala que va de 1 (totalmente en desacuerdo) a 7 (totalmente de acuerdo). Busca establecer la medida en que una persona está abierta al cuestionamiento y la búsqueda de sentido en su vida. Para esta investigación se ha obtenido un índice de consistencia interna de 0,57.

\section{PROCEDIMIENTOS}

El estudio se diseñó en concordancia con los criterios establecidos por el equipo internacional. En un primer momento, el equipo de investigación costarricense conformado por tres psicólogos y un sociólogo, se encargaron de la traducción de todos los instrumentos, así como de aplicar el procedimiento de traducción inversa, el cual resultó adecuado. Posteriormente, se llevó a cabo una sesión de grupo focal con estudiantes universitarios como los que se buscaba entrevistar, con el fin de evaluar la comprensión de los instrumentos y determinar si existían anomalías. Luego, un grupo de cinco asistentes de investigación, se encargaron de la aplicación de los instrumentos en la Sede Central de la Universidad de Costa Rica. En un momento final, estos asistentes de investigación se encargaron de realizar la digitación de los resultados, para conformar las bases de datos en el SPSS-PC para Windows, a partir de lo cual se han llevado a cabo diversos análisis estadísticos que se presentan en el siguiente apartado. 


\section{RESULTADOS}

A continuación se describe, en primer lugar, las características psicosociales y psicoreligiosas del grupo de jóvenes en estudio. En segundo lugar, se buscará mostrar en qué medida el fundamentalismo religioso presente, podría eventualmente condicionar las características socioemocionales y la satisfacción con la vida, por una parte. Por otra parte, cómo el fundamentalismo religioso podría condicionar la religiosidad en general, la confianza en las instituciones y el autoritarismo.

\section{CARACTERÍSTICAS PSICOSOCIALES}

Este estudio se ha enfocado en cuatro características psicosociales, a saber, el autoritarismo de derecha, la orientación e intereses políticos, el afecto y la satisfacción con la vida (ver Cuadro 1).

CUADRO 1

ESTADÍSTICOS DESCRIPTIVOS BÁSICOS EN LAS DIMENSIONES PSICOSOCIAL Y PSICORELIGIOSA

\begin{tabular}{|c|c|c|c|c|c|}
\hline $\mathrm{N}=184$ & $M$ & $D S$ & $\begin{array}{l}\text { Mínimo } \\
\text { Máximo }\end{array}$ & $\begin{array}{c}\text { Alpha } \\
\text { de Cronbach }\end{array}$ & $\begin{array}{l}\text { Número de } \\
\text { reactivos }\end{array}$ \\
\hline \multicolumn{6}{|l|}{ DIMENSIÓN PSICORELIGIOSA } \\
\hline Búsqueda existencial & 38,59 & 8,22 & $16-57$ & 0,57 & 9 \\
\hline Práctica religiosa & 14,39 & 5,62 & $3-21$ & 0,82 & 3 \\
\hline Cuatro polos religiosos & 41,22 & 19,48 & $12-84$ & $0,84 / 0,88 / 0,87 / 0,85$ & 12 \\
\hline Fundamentalismo religioso & 35,09 & 15,00 & $12-78$ & 0,86 & 12 \\
\hline \multicolumn{6}{|l|}{ DIMENSIÓN PSICOSOCIAL } \\
\hline Autoritarismo de derecha & 46,86 & 8,18 & $27-68$ & $0,57 / 0,58 / 0,59$ & 12 \\
\hline Orientación e intereses políticos & 16,99 & 8,87 & $00-42$ & 0,84 & 5 \\
\hline Afecto positivo & 28,59 & 4,27 & $14-42$ & 0,74 & 5 \\
\hline Afecto negativo & 10,80 & 3,16 & $5-22$ & 0,75 & 5 \\
\hline Satisfacción con la vida & 25,94 & 6,22 & $5-35$ & 0,87 & 5 \\
\hline
\end{tabular}

Fuente: Elaboración propia.

Entre los resultados más significativos se puede mencionar el hecho según el cual, en esta muestra de jóvenes universitarios, la característica autoritaria de derecha con mayor puntaje promedio es el conservadurismo con 15,96 (DS= 5,41; Var= 29,28; Min-Máx= 4-28); posteriormente se sitúa la agresividad con 11,30 (DS= 4,58; Var= 21,04; Min-Máx= 3-21) y finalmente la sumisión con 9,18 (DS= 3,66; Var= 13,42; Min-Máx= 3-20). Cabe señalar que las diferencias entre estos tres aspectos del autoritarismo de derecha, son estadísticamente significativas (agresividad $\mathrm{t}=33,42, \mathrm{gl}=183$, sig-bi $=, 000$; sumisión $\mathrm{t}=$ 33,91, gl= 182, sig-bi $=, 000$ y conservadurismo $\mathrm{t}=39,80, \mathrm{gl}=181$, sig-bi=, 000$)$. En cuanto a las diferencias por género respecto a estas dimensiones, se encuentra que no existe diferencia significativa entre hombres y mujeres referente a las subescalas de sumisión $(t=1,91$; gl= 119,07; sig-bi= ,059) y conservadurismo $(t=$ ,799; gl= 117,58; sig-bi= ,43). Para la escala de agresividad, sí existen diferencias significativas por género $(t=3,29$; gl=127,62; sig-bi $=, 001)$, según la cual los varones obtienen un puntaje más alto en agresividad. 
También se ha realizado una evaluación del afecto, cuyo puntaje promedio para el afecto positivo es de 28,59 (DS= 4,27; $\operatorname{Var}=18,20$; Min-Máx=14-42). En cuanto al afecto negativo se ha obtenido un puntaje promedio mucho más bajo, de 10,80 (DS= 3,16; Var=9,98; MinMáx= 5-22). Como parece claro, las diferencias entre estas dos subescalas son estadísticamente significativas (afecto positivo $\mathrm{t}=90,17, \mathrm{gl}=$ 180 , sig-bi $=, 000$; afecto negativo $\mathrm{t}=46,12, \mathrm{gl}=$ 181 , sig-bi $=, 000$ ), predominando en la muestra el afecto positivo. Al mismo tiempo que esta muestra es más positiva en cuanto al afecto, también parece estar más satisfecha con la vida que insatisfecha, ya que el puntaje promedio de 25,94 se encuentra más cerca de su puntaje máximo (DS= 6,22; Var= 38,63; Min-Máx= 5-35). Además, la satisfacción con la vida no muestra diferir en cuanto a hombres y mujeres para la muestra en cuestión ( $\mathrm{t}=, 008, \mathrm{gl}=173$, sig-bi= ,99).

La confianza en instituciones $y$ organizaciones tales como la policía, los políticos, los partidos políticos y la Organización de las Naciones Unidas también fue evaluada. Obtuvimos un puntaje promedio de 16,99 de confianza (DS= 8,87; Var= 78,71; Min-Máx= 0-42), apenas por encima de la mediana $(16,0)$ y mucho más cerca del puntaje mínimo con una varianza muy grande, todo lo cual nos confirma, una vez más, el bajo nivel de confianza existente entre las personas jóvenes en esas instituciones importantes para la gobernanza. Dichas personas manifiestan que en un continuum entre pertenecer a una orientación política de derecha o de izquierda, el 35,7\% manifiesta tener una orientación hacia la izquierda del punto medio, mientras que el $32,1 \%$ se ubican hacia la derecha del punto medio. El 35,3\% se ubica justo en el centro del continuum.

\section{CARACTERÍSTICAS PSICORELIGIOSAS}

Enfocados en la religiosidad de la muestra (ver Cuadro 1), se plantea su descripción a través de un examen de los cuatro polos fundamentales de la religiosidad, las prácticas religiosas, el fundamentalismo religioso y la búsqueda existencial. Los cuatro polos fundamentales de la religiosidad incluyen cuatro componentes: a) las creencias, b) los rituales $y$ emociones manifiestas, c) las reglas morales $y$ d) el sentido de comunidad y agrupación. El elemento con mayor puntaje promedio es el de las reglas morales, el cual se refiere al comportamiento según reglas éticas y morales, con 10,98 (DS= 5,35; $\operatorname{Var}=28,64$; Min-Max= 3-21). Luego sigue el componente de las creencias, entendidas como creencia y búsqueda de significado de la vida con 10,77 (DS= 5,38; Var= 28,92; Min-Max= 3-21). Posteriormente, el sentido de comunidad y agrupación, esto es, el sentido de comunidad $y$ pertenencia al grupo con 9,84 (DS= 5,35; Var= 28,64; Min-Max= 3-21). Por último, los rituales, incluyendo también la estética religiosa y las emociones involucradas en los rituales, cuyo puntaje promedio fue de 9,7 (DS= 5,39; Var= 29,07; Min-Max= 3-21). Al evaluar potenciales diferencias de género, no se encuentran diferencias estadísticamente significativas para ninguno de estos cuatro componentes.

En cuanto a las diferencias entre las subescalas, el componente de las creencias difiere significativamente de los componentes de sentido de comunidad e identidad $(t=2,33 ; \mathrm{gl}=180$; sig-bi=,02) y de estética, emotividad y rituales $(t=2,86 ; g l=180 ;$ sig-bi=.01), no siendo así para el componente de reglas éticas y morales $(t=0,51$; gl=180; sig-bi=,61). El componente de sentido de comunidad y pertenencia al grupo difirió de manera significativa del componente de reglas éticas y morales $(t=2,73$; gl=180; sig-bi=,01) y del significado de la vida $(t=2,30 ; g l=178$; sig$\mathrm{bi}=, 02)$, sin embargo, no difiere significativamente del componente de estética, emotividad y rituales $(t=0,35 ; g l=180$; sig-bi=,72).

Respecto a las características del fundamentalismo religioso, se encuentra una puntuación promedio de 35,09 (DS= 15; Var= 225,07; Min-Max= 12-78), ubicándose por debajo de la puntuación central (42). Esto indica que existen bajos niveles de fundamentalismo en ambos géneros, dándose un pequeño aumento en las mujeres $(35,70$; DS $=15,44)$ respecto a los 
hombres $(34,50$; DS $=14,86)$, no siendo esta diferencia estadísticamente significativa $(t=-0,47$; $\mathrm{gl}=101,3$; sig-bi= 0,635).

En cuanto a las prácticas religiosas, la puntuación promedio es de 14,39 (DS=5,62; Min-Max=3-21), revelando una puntuación media por encima del puntaje central $(10,5)$, dándose en mayor medida en mujeres $(15,66$; $\mathrm{DS}=5,19)$ que en hombres $(13,72 ; \mathrm{DS}=5,73)$, siendo dicha diferencia estadísticamente significativa $(t=2,26 ; \mathrm{gl}=127,27$; sig-bi= .025). En cuanto a los tres componentes considerados dentro de las prácticas religiosas, la importancia de Dios en sus vidas obtuvo 5,31 mientras que la importancia de la espiritualidad obtuvo un puntaje de 5,25 y finalmente, la importancia de la religión para la vida de estas personas jóvenes tuvo una puntuación promedio de 3 ,83. Solo la importancia de Dios $(t=11,4 ; \mathrm{gl}=$ 183; sig-bi $=, 000)$ y de la espiritualidad $(t=$ $11,67 ; \mathrm{gl}=182$; sig-bi $=, 000)$ muestran alejarse significativamente de la puntuación media $(3,5)$, mientras que la importancia de la religión para la vida de los participantes no parece alejarse significativamente del punto medio $(t=1,94 ; \mathrm{gl}=184$; sig-bi= 054$)$. No existen diferencias significativas entre el género de los participantes y ninguno de los componentes. Esta tendencia de preferencias: Dios (el objeto trascendente), espiritualidad, religión, se relaciona con los hallazgos para la juventud costarricense en general.

En lo referente a la búsqueda existencial, entendida como la disponibilidad para comprometerse en un proceso de cuestionamiento sobre el significado de la vida y su visión de mundo, se encuentra un puntaje promedio de 38,59 (DS= 8,22; Var=67,64; Min-Max= 16-57), siendo su diferencia respecto al punto central de la escala (36), estadísticamente significativa $(t=4,27$; $\mathrm{gl}=183$; sig-bi=,000). Si bien, los jóvenes varones obtuvieron un puntaje promedio mayor (39, 27 ; $\mathrm{DS}=7,96)$ respecto a las mujeres $(37,60$; $\mathrm{DS}=8,22$ ), estas diferencias no fueron estadísticamente significativas $(t=1,29 ; \mathrm{gl}=113,18$; sig-bi=0,200).

\section{ANÁLISIS DE ASOCIACIÓN Y VARIABLES DE PREDICCIÓN}

A continuación se examinan las asociaciones más importantes entre las variables en estudio. En primer lugar, se brindará una breve perspectiva del análisis que se realizó a partir de la escala RWAS como unitaria, a partir del análisis de factores realizado. A pesar de que esta no será la perspectiva general que tomará el presente artículo, se realizará una breve descripción de sus resultados. Posteriormente, se presenta con base en este análisis, las variables que se logran extraer como predictores del fundamentalismo religioso en esta muestra de jóvenes universitarios.

Al utilizar la escala RWAS como unitaria, se realizó un análisis de Regresión Múltiple mediante el método Enter con las mismas variables que se utilizaron como predictores en el modelo principal. Sobre la variable de fundamentalismo religioso, el mejor predictor fue la subescala de rituales de la escala de FPRS, con un $\beta=, 34 ; t(162)=$ 5,$39 ; \mathrm{p}<, 001$, seguida por el autoritarismo de derecha (RWAS como escala completa) con un $\beta=, 31 ; t(162)=5,5 ; \mathrm{p}<, 001$, el rezar con un $\beta=, 28 ; t(162)=4,31 ; \mathrm{p}<, 001$ y búsqueda existencial con un $\beta=-, 14 ; t(162)=2,6 ; \mathrm{p}<, 05$. De este análisis se obtuvo un $\mathrm{R}^{2}$ corregida $=, 54$; $F(2,162)=49,12 ; \mathrm{p}<, 001$.

Tal como se verá más adelante, la dirección y fuerza de las variables utilizadas en el modelo principal no difieren en gran medida del modelo alternativo planteado a partir de la escala RWAS como unitaria. Al contrario, el aporte a la explicación de la varianza que la escala RWAS completa aporta es mucho menor que la que se obtiene en el modelo principal. Esto implica que, a pesar de que el modelo utiliza una subescala con una consistencia interna baja, la utilización de la escala completa (que cuenta con un alpha de Cronbach aceptable) muestra un menor peso como predictora. Esto debido probablemente a que tres de las cuatro subescalas de RWAS no mostraron ser buenas predictoras en el modelo principal, lo que sería consistente con el 
hecho de que la reducción de la varianza explicada por la escala completa se deba a la diseminación de la varianza compartida entre esta escala $y$ la de fundamentalismo religioso dentro del conjunto de las cuatro subescalas.

Lo que es importante señalar es que, a pesar de realizar el análisis con la escala completa de RWAS, los resultados son consistentes con los resultados del análisis principal. Si bien, son una deficiencia los bajos índices de consistencia interna en esta escala, la posibilidad de vislumbrar resultados similares utilizando como predictora a la escala unitaria de autoritarismo de derecha permite abordar la teorización cautelosa, a partir de los resultados de la regresión del modelo utilizado.

Para el modelo principal, las variables independientes asociadas con significancia estadística con la variable dependiente fundamentalismo religioso, se ha encontrado que dos características del autoritarismo de derecha como son la sumisión y el conservadurismo, se asocian significativa y positivamente con dicha variable dependiente. Sin embargo, de estas dos variables independientes, parece más importante el conservadurismo que la sumisión. Por otro lado y como es esperable, el fundamentalismo religioso y la búsqueda existencial están asociadas inversamente de manera significativa. Por otro lado, las prácticas religiosas también están asociadas positivamente $y$ de forma significativa con el fundamentalismo religioso, al igual que el sentido de comunidad y las reglas ético-morales, pero estas poseen una asociación más alta que el sentido de comunidad. Con este análisis de asociación, se puede observar una tendencia a partir de la cual, tanto el conservadurismo como las reglas morales, adquieren mayor relevancia para el fundamentalismo religioso. 


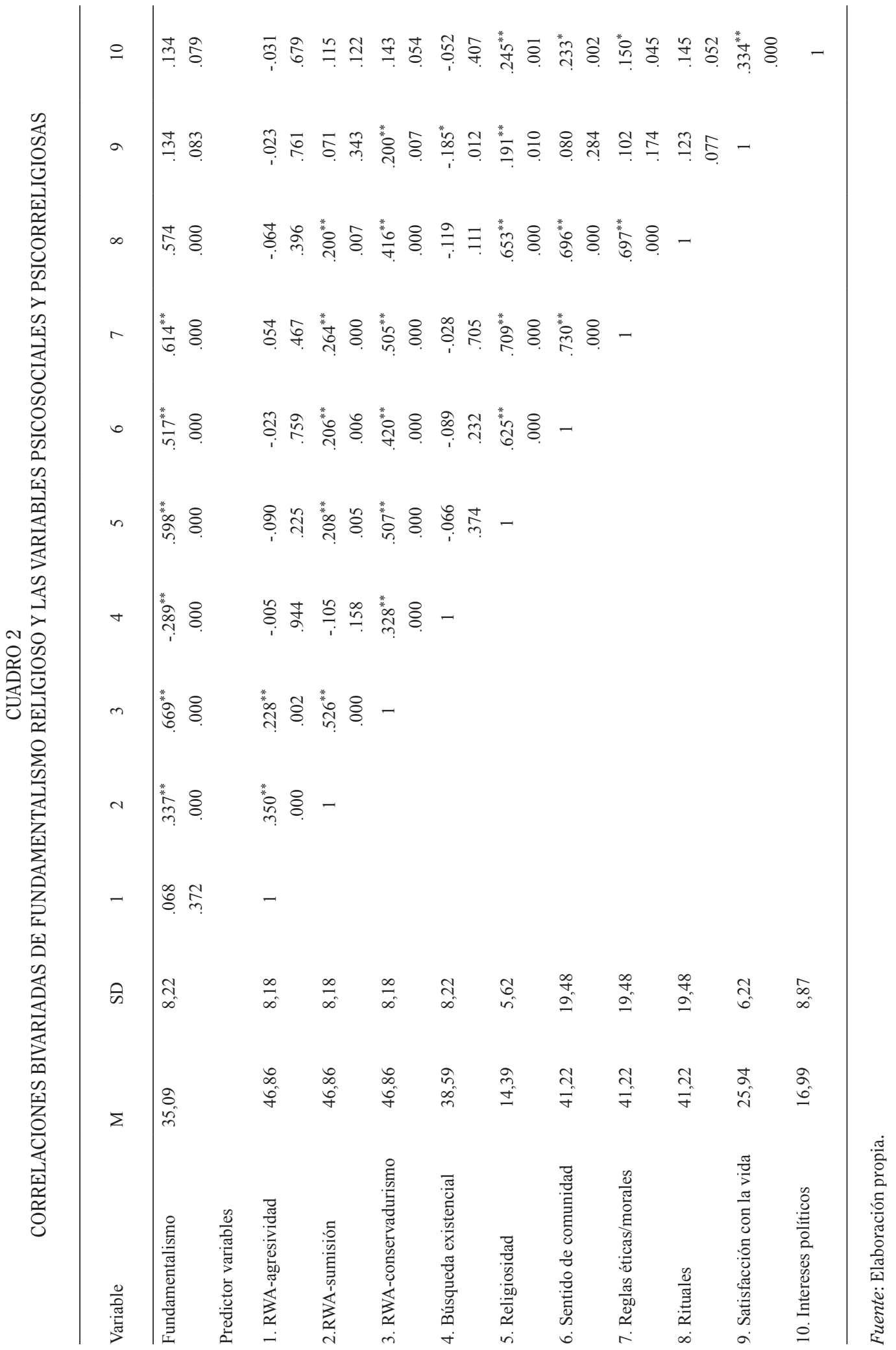


Para contar con una evidencia añadida al análisis de asociación mediante el análisis de correlación bivariado, se ha efectuado también un análisis de regresión lineal jerárquica. De esta manera, se estudia el patrón de efectos de predicción. Así, después de evaluar si estaba presente en los datos alguna violación de los supuestos requeridos para ejecutar los análisis estadísticos (normalidad, linealidad, homocedasticidad, valores atípicos) y obteniendo resultados satisfactorios para cada uno de estos supuestos, es posible examinar los resultados del análisis de regresión resumidos en el Cuadro 3. Este análisis de regresión ha permitido despejar las variables independientes significativas, que conforman una configuración con el fundamentalismo religioso, a saber $y$ en orden de importancia el conservadurismo de derecha, la práctica religiosa de rezar, la ritualidad emotiva $y$ de manera inversa, la cuestión de la búsqueda existencial. Así pues, a mayores niveles de búsqueda existencial, menores niveles de fundamentalismo religioso. El modelo de regresión con el cual se despejan estas variables explica el $66 \%$ de la varianza. Además, este modelo permite confirmar el análisis de asociación bivariado y despeja las variables independientes no significativas.

CUADRO 3

RESUMEN DEL ANÁLISIS DE REGRESIÓN PARA LAS DIMENSIONES PSICOSOCIALES Y PSICORELIGIOSAS QUE PREDICEN EL FUNDAMENTALISMO RELIGIOSO

\begin{tabular}{lccc}
\hline VARIABLE DEPENDIENTE & \multicolumn{2}{c}{ FUNDAMENTALISMO RELIGIOSO } \\
\hline VARIABLES INDEPENDIENTES & $\mathrm{B}$ & SEB & $0,71 \mathrm{a}$ \\
\hline Conservadurismo & 1,95 & 0,16 & $0,36 \mathrm{~b}$ \\
Rezar & 2,53 & 0,41 & $0,24 \mathrm{c}$ \\
Rituales & 0,68 & 0,17 & $-1,94 \mathrm{~d}$ \\
Búsqueda Existencial & $-0,37$ & 0,09 & \\
\hline
\end{tabular}

Fuente: Elaboración propia.

Nota: Fundamentalismo religioso: $(\mathrm{N}=158), \mathrm{R}^{2}=66 \% \mathrm{~F}(1,149)=15,849 ; p=.000,{ }^{\mathrm{a}} p=.000,{ }^{\mathrm{b}} p=.000,{ }^{\mathrm{c}} p=.000,{ }^{\mathrm{d}} p=.000$

\section{DISCUSIÓN}

El interés de esta investigación se ha concentrado en describir las características psicosociales y psicoreligiosas de una muestra de jóvenes universitarios. Esto ha permitido posteriormente examinar cómo se configura el fundamentalismo religioso con algunas dimensiones relevantes de tipo socio-cognoscitivas y psicoreligiosas entre estos jóvenes universitarios.

Al considerar más específicamente los resultados de las dimensiones psicosociales, es posible establecer que en esta muestra hay una tendencia al conservadurismo de derecha, así como poca confianza en importantes instituciones sociopolíticas, en un contexto de indefinición de los y las jóvenes entre la izquierda y la derecha como tendencias políticas. Aún así, este hallazgo no puede hacernos pensar en ninguna certeza al respecto, pues los índices de consistencia interna del instrumento de autoritarismo de derecha son bajos. Esta es una importante limitación de nuestro estudio en cuanto a establecer este hallazgo de una manera bien contrastada y segura como tal.

Al hallazgo anterior, cabe añadir que se observa a los jóvenes con una tendencia mayor a ser agresivos, mientras que las jóvenes no. A esta tendencia, que se puede situar en el nivel de una representación colectiva, la acompaña otra más individual a sentir mayor satisfacción con la vida $y$ con la predominancia del afecto positivo en su estado de ánimo. Sin embargo, este último aspecto refleja más claramente el estado de ánimo durante la aplicación de los instrumentos. También en este caso de la agresividad, 
es importante considerar los hallazgos como tentativos o limitados, pues el instrumento de medición correspondiente expresa un índice de consistencia interna bajo.

Que este grupo de jóvenes aparezca con puntajes altos en el conservadurismo de derecha, no es sorprendente, pues este hallazgo confirma otros análisis provenientes de la literatura sociológica costarricense enfocada sobre el análisis de cuestiones político-ciudadanas en general. Coincide pues con la imagen de una sociedad costarricense conservadora en sus opciones políticas, religiosas y en su visión de mundo. Por un lado, la desconfianza en las instituciones es una cuestión persistente en las encuestas de opinión y en el análisis sociopolítico, cuya base empírica es precisamente el desencanto de la población en general y de la juventud en particular, respecto de los grupos políticos tradicionales $y$ de las formas convencionales de gestión del poder, las cuales están más al servicio de intereses personales y mucho menos al servicio del interés general de la sociedad. Sin embargo, como se señaló antes; para interpretar la dimensión de conservadurismo se requiere mucha cautela, dado el índice de consistencia interna obtenido.

Se encontró una importante dificultad con las hipótesis que buscan hacer una conexión entre los fenómenos religiosos y los fenómenos políticos, las que podrían derivarse de las dificultades metodológicas, pero sin duda también comportan dificultades sustantivas ligadas a la teorización en las que se basan las hipótesis. Por ejemplo, Rodríguez (2007) analiza que el fundamentalismo no se comporta como predictor significativo de nacionalismo porque implica una forma de relación con la creencia religiosa que se encuentra "por encima" de otras vinculaciones posibles, entre ellas el discurso nacionalista (Rodríguez: 187). La metáfora de ponerse por encima por parte del fundamentalista religioso parece plausible, pero resulta contradictoria con las características del fundamentalismo religioso que paulatinamente la literatura especializada dilucida. Por ejemplo, parece más plausible la operatividad del principio de intra-textualidad en el fundamentalismo, según el cual la apropiación del texto sagrado y su interpretación intra-textual conducen al fundamentalista a descubrir verdades absolutas no negociables que están más allá de la persona como una base de realidad objetiva, contra la cual la persona da un sentido a su vida e interpreta el significado de las experiencias personales (Muluk y Sumaktoyo, 2010). Más que una metáfora de ir por encima del sistema de creencias, en el fundamentalismo la metáfora de ir hacia dentro parece más explicativa. De ahí resulta posible ser fundamentalista $y$ ser prosocial como demuestran Blogowska y Saroglou (2011), lo cual podría conducir a una relación un tanto distinta con los objetos políticos como el nacionalismo o incluso con el conservadurismo de derecha.

Ahora bien, las tendencias individuales hacia la auto-satisfacción vital y el estado de ánimo positivo en esta muestra, provienen de la adscripción a la clase media (alta $y$ media), por parte del estudiantado de la universidad en la que se ha realizado el estudio, la cual se autopercibe de manera positiva. Es una imagen que coincide con la auto-percepción positiva de la situación personal, como un universo desvinculado o fragmentado respecto al universo social y político de la sociedad y la cultura costarricense. Como se indicó anteriormente, si se examinan los aspectos predominantes en la visión de mundo de la juventud costarricense, a saber, la familia y Dios como elementos articuladores de un sentido para la vida, es comprensible que también entre jóvenes universitarios aparezcan tendencias similares, mostrando así que la intensidad del cambio social podría no ser tan vertiginosa como se piensa en algunas ocasiones (Consejo Nacional de Política Pública de la Persona Joven, 2008). Con esto no se quiere decir que no haya una tendencia de cambio, sino solamente se sugiere como la velocidad del cambio psicosocial y psicoreligioso en este caso, no parece ser como algunas veces suponemos en la teoría social o en las ciencias sociales. Sin duda, en la muestra estudiada hay un margen de indeterminación en cuanto a la afiliación religiosa se refiere. Aún así, esto no impide establecer cuanto aparece como una evidencia en nuestra observación, pues efectivamente, aún cuando la afiliación religiosa al cristianismo entre la juventud $y$ en general 
tiende a disminuir, en esta muestra específica dicha tendencia se mantiene aún cuando parece configurarse una afiliación religiosa mayoritaria al cristianismo. Simultáneamente, esta tendencia relativa observada parece confirmarse en algún sentido, pues como se sabe, la juventud costarricense en general manifiesta una tendencia correlativa con la menor participación en actividades socio-políticas y en el activismo social (Consejo Nacional de Política Pública de la Persona Joven, 2008), lo cual es en sí desalentador en cuanto implica un abandono del espacio de lo público ahora y quizás en el futuro cercano. No obstante, dicha interpretación podría modificarse con las próximas encuestas por realizar en el país y los nuevos datos, lo que no haría sino confirmar el supuesto según el cual hay una tendencia de cambio en el tiempo.

A la misma vez, al considerar los resultados de las dimensiones psico-religiosas, los datos señalan que esta muestra de jóvenes es religiosa practicante, en cuanto Dios y la espiritualidad son importantes para dar sentido a su vida. No obstante, las instituciones religiosas no gozan de esta importancia. Ahora bien, las mujeres son más practicantes que los varones, lo cual es un hallazgo persistente al menos en el ámbito internacional (Lippman y Dombrowski, 2006). Esto se complementa con una aspiración a comportarse de acuerdo con un conjunto de reglas éticas y morales, vinculadas con las creencias religiosas en particular. Se trata de contenidos morales en una perspectiva religiosa. Por otro lado, se valora el sentido de comunidad junto con los rituales, pero en menor medida, lo cual es coherente con la idea de una espiritualidad sin religión-institución, pero sí con valores y creencias.

Sin embargo, esta configuración de lo religioso cabe entenderla junto al hecho que esta muestra expresa un bajo nivel de fundamentalismo religioso y una apertura clara al cuestionamiento de sus propias visiones de mundo y del significado de la vida. En consecuencia, esta condición de la muestra estudiada, el bajo nivel de fundamentalismo religioso, es muy importante. Supone la existencia de aspectos comúnmente atribuidos al fundamentalismo religioso como un muy alto nivel de autoritarismo de derecha o un bajo nivel de búsqueda existencial, efectivamente presentes en los resultados encontrados, aunque no de manera significativamente alta. Pero además, implica que se sitúa dentro de los rangos habituales de la adhesión a valores y actitudes religiosas, sin ser fundamentalista, lo cual resulta ser una tendencia predominante entre la juventud costarricense al coincidir estos resultados con aquellos de la Encuesta Nacional de Juventudes. También con estos resultados se logra perfilar otra tendencia relevante: efectivamente, no solo el nivel de fundamentalismo religioso es bajo, sino que cada vez la menor importancia ofrecida a las instituciones religiosas o a lo religioso instituido se pone de manifiesto aquí, subrayando simultáneamente la importancia atribuida a una determinada representación de Dios y a la espiritualidad. De ahí es posible comprender cómo la importancia concedida a la práctica de rezar, teniendo este contexto como trasfondo, se orienta mucho más al desarrollo de una espiritualidad personal y mucho menos a la adhesión a una creencia, sostenida o respaldada por la adhesión a lo religioso instituido. Solo en el marco de una espiritualidad personal, desprovista de las normas en las cuales la religión-institución exige convergencia, es posible entonces la apertura cognitiva suficiente, capaz de posibilitar el cuestionamiento del sentido de la vida y la ampliación de la búsqueda de opciones existenciales. Con esto afirmamos que en el fundamentalismo religioso, tal apertura cognoscitiva no sería posible. Se puede establecer que, al contrario del fundamentalismo religioso, se está asumiendo la religiosidad, pero re-interpretada y re-integrada, como una opción espiritual personal (Petersen, 2008). Sin embargo, esto no implica, según lo observado, renunciar a valores $y$ actitudes orientadoras para estas personas jóvenes, hacia el conservadurismo socio-político y socio-moral.

La ventaja de contar con datos cuya dinámica es relativamente autónoma de las condiciones del sujeto cognoscente, consiste en que se puede operar con una expectativa de objetivación de mayor confianza, pero eso sí, ni absolutista ni fetichista del método. Así las cosas, cabe admitir la condición dinámica y 
cambiante de los procesos psicosociales y psicoreligiosos relacionados con el fundamentalismo religioso, pero esto no acontece necesariamente en la dirección con frecuencia esperada por las expectativas y deseos de las ciencias sociales $y$ de la Psicología, lo cual implica reconocer la autonomía de los procesos psicosociales respecto a la mirada del científico como observador. En efecto, se cuenta con evidencias para pensar que la juventud costarricense tiende al conservadurismo político, socio-moral y religioso, pero con los matices señalados. Esto es relevante para el futuro de la sociedad costarricense, la cual más tarde que pronto, podría llegar a situarse algún día en un punto de convergencia con las orientaciones políticas, religiosas y morales que buscan una mayor predominancia del pluralismo político-ideológico, el relativismo moral y la tolerancia espiritual universal.

Unas condiciones socio-culturales de este tipo, quizás caen detrás del punto de llegada de otras sociedades como las post-industriales, porque en Costa Rica las condiciones de la racionalidad que provocan el desarrollo científico-tecnológico y la explotación intensiva de los recursos naturales, no es aún completa. La hipótesis aquí sería que las condiciones de la racionalidad, disminuyen su fuerza - no ética- al universo religioso-espiritual, retrayéndolo y dando lugar así a mayor pluralismo político-ideológico, mayor relativismo moral y mayor tolerancia espiritual-religiosa. Más bien, las energías sociales puestas en la expectativa positiva de un mundo mejor, mediante los esfuerzos del cambio cultural que representa la conservación, la protección y la sostenibilidad ambiental, parecen traer consigo unas actitudes y una visión de mundo "espiritual" ligada a la contemplación de la naturaleza, la cual si bien sería una "espiritualidad" alejada de cualquier institucionalidad conservadora, constituye una expectativa normativa y teleológica, una confianza en una energía trascendente inmanente, pretendiendo articular las transformaciones psicosociales necesarias y perpetuar la nueva cultura de preservación del planeta.

Para finalizar, cabe señalar dos limitaciones claras del presente estudio. Una primera limitación se deriva de los bajos índices de consistencia interna alpha de Cronbach para las sub-dimensiones de búsqueda existencial $y$ autoritarismo de derecha. Esta limitación convierte algunas de nuestras inferencias en arriesgadas $y$ deben ser tomadas en cuenta en los casos específicos antes comentados, con muchísima cautela, pues podrían ni siquiera señalar una tendencia. Superar esta dificultad requiere la realización de estudios con muestras más numerosas, lo cual permitiría reducir el efecto del tamaño, situación a la cual parece ser muy sensible el alpha de Cronbach. Cabe señalar que las deficiencias en la traducción de las escalas, así como, las características propias de la muestra en cuestión pudieron haber contribuido a dichas problemáticas.

En segundo lugar, el estudio no contrasta a esta muestra de universitarios con una muestra de personas universitarias o no, quienes reúnan la condición de ser claramente religiosas o adscritas a una denominación fundamentalista. En próximas investigaciones estas dificultades habrán de preverse con el fin de evitar los límites que se imponen a las inferencias y limitaciones actuales.

\section{REFERENCIAS}

LIBROS

Hood, R.W.; Hill, P.C. $y$ Williamson, W.P. The psychology of religious fundamentalism. New York: Guilford Press, 2005.

Kirkpatrick, L.A. "Evolutionary psychology: an emerging new foundation for the psychology of religion". Handbook of the psychology of religion and spirituality. Paloutzian, R.F. y Park, C.L. (eds.). New York: Guilford Press, 2005.

Lippman, L.H. y Dombrowski-Keith, J. "The demographics of spirituality among youth: international perspectives". The handbook of spiritual development in childhood and adolescence. Roehlkepartain, E.C.; King, P.B.; Wagener, L. y Benson, P.L. (eds.). USA. Sage, 2006: 109-123.

Mayers, L.S.; Gamst, G. y Guarino, A.J. Applied multivariate research: design and interpretation. USA: Sage, 2007. 
Schäfer, H. Protestantismo y crisis social en América Central. Departamento Ecuménico de Investigaciones DEIUniversidad Luterana Salvadoreña, 1992.

Tabachnik, B.G. y Fidell, L.S. Using multivariate statistics. $2^{\text {nd }}$ ed. USA: Pearson, 2006.

\section{REVISTAS}

Altemeyer, B. "Why do religious fundamentalists tent to be prejudices?". The International Journal for the Psychology of Religion 13 (1). 2003: 17-28.

Altemeyer, B. y Hunsberg, B. "Authoritarianism, religious fundamentalism, quest and prejudice". The International Journal for the Psychology of Religion 2 (2). 1992: 113-133.

Altemeyer, B. y Hunsberger, B. "A revised religious fundamentalism scale: the short an sweet of it". International Journal for the Psychology of Religion 14. 2004: 47-54.

Blogowska, J. y Saroglou, V. "Religious fundamentalism and limited prosociality as a function of the target". Journal for the Scientific Study of Religion 50 (1). 2011: 44-60.

Diener, E.; Emmons, R.A.; Larsen, R.J. y Griffin, S. "The satisfaction with life scale". Journal of Personality Assessment 49. 1985: 71-75.

Funke, F. "The dimensionality of rightwing authoritarianism: lessons from the dilemma between theory and measurement". Political Psychology 26. 2005: 195-218.

Galen, L.W.; Wolfe, M.B.; Deleeuw, J. y Wyngarden, N. "Religious fundamentalism as schema: influences on memory for religious information". Journal of Applied Social Psychology 39 (5). 2009: 1163-1190.

Gosling, S.D.; Rentfrow, P.J. y Swann Jr., W.B. "A very brief measure of the big-five personality domains". Journal of Research in Personality 37. 2003: 504-528.

Gribbins, T. y Vandenberg, B. "Religious fundamentalism, the need for cognitive closure, and helping". The International Journal for the Psychology of Religion 21. 2011: 106-114.

Jakubowska, U. y Oniszczenko, W. "The role of personality, cognitive, environmental and genetic factors as determinants of religious fundamentalism: a twin study in a polish sample". Studia Psychologica 52 (3). 2010: 253-263.

Kinnval, C. "Globalization and religious nationalism: self, identity, and the search for ontological security". Political Psychology 25 (5). 2004: 714-767.

Muluk, H. y Sumaktoyo, N.G. "Intratextual fundamentalism and the desire for simple cognitive structure: the moderating effect of the ability to achieve cognitive structure". Archive for the Psychology of Religion 32. 2010: 217-238.

Núñez-Alarcón, M.; Moreno-Jiménez, M.P. y Moral-Toranzo, F. "Modelo causal del prejuicio religioso". Anales de Psicología 27 (3). 2011: 852-861.

Petersen, A.C. "Spiritual development in adolescence: toward enriching theories, research, and professional practice". New Directions for Youth Development: Special Issue: Spiritual Development 118. 2008: 119-130.

Rodríguez, J.M. "La religión como predictor de actitudes hacia la Nación". Actualidades en Psicología 21. 2007: 167-191.

Saroglou, V. "Beliving, bonding, behaving, and belonging: the big four religious dimensions and cultural variation". Journal of Cross-Cultural Psychology 20 (10). 2011: 16-22.

Saroglou, V. y Muñoz-García, A. "Individual differences in religion and spirituality: an issue of personality traits and/or values". Journal for the Scientific Study of Religion 47 (1). 2008: 83-101.

Solís, M. "Microsociología de la interacción y constitución del sí mismo en la vivencia comunitaria neopentecostal". Actualidades en Psicología 22. 2008: 19-42.

Thompson, E.R. "Development and validation of an internationally reliable short- 
form of the Positive and Negative Affect Schedule (PANAS)". Journal of CrossCultural Psychology 38 (2). 2007: 227242.

Van Pachterbeke, M.; Keller, J. y Saroglou, V. "Flexibility in existential beliefs and worldviews: introducing and measuring existential quest". Journal of Individual Differences. In press.

Watson, P.J.; Chen, Z. y Hood, R.W. "Biblical foundationalism and religious reflection: polarization of faith and intellect oriented epistemologies within a Christian ideological surround". Journal of Psychology and Theology 39 (2). 2011: 111-121.
OTROS

Consejo Nacional de Política Pública de la Persona Joven. Primera Encuesta Nacional de Juventud. Principales resultados. Costa Rica 2008. Viceministerio de Juventud, Fondo de Población de las Naciones Unidas. 2008.

Halman, L. The European value study: a third wave. Source book of the 1999/2000 European Value Study Surveys. Tilburg, NL. EVS-WORC-Tilburg University, 2001.

Fecha de ingreso: 28/03/2012 Fecha de aprobación: 19/07/2012 


\section{APÉNDICE 1}

\section{ESCALA DE AFECTO POSITIVO Y NEGATIVO}

The International Positive and Negative Affect Schedule Short Form, I-PANAS-SF (Thompson, 2007).

Pensando en usted mismo/a y en cómo normalmente se siente, con qué frecuencia usted se siente:

1. Disgustado/a

2. Hostil

3. Alerta

4. Avergonzado/a

5. Inspirado/a

6. Nervioso/a

7. Determinado/a

8. Atento/a

9. Asustado/a

10. Activo/a

\section{ESCALA DE AUTORITARISMO DE DERECHA}

Right-Wing Authoritarianism Scale (Funke, 2005).

Por favor encierre en un círculo el número del 1 al 7 que mejor indica su grado de acuerdo o desacuerdo con cada afirmación.

1. Lo que nuestro país realmente necesita, en vez de más derechos, es una buena dosis de leyes $y$ orden.

2. Lo que nuestro país realmente necesita es un/a presidente fuerte $y$ determinado/a, que acabe con la maldad $y$ nos devuelva al buen camino.

3. No hay ningún crimen que justifique la pena de muerte.

4. Es importante proteger, a como de lugar, los derechos de los/as extremistas y de las personas que se salen de la norma.

5. La obediencia y el respeto a la autoridad son los valores más importantes que los/ as niños/as deben aprender.

6. Las verdaderas claves para una "vida buena" son la obediencia, la disciplina $y$ la virtud.

7. Los días en los que las mujeres estaban sometidas deben quedar estrictamente en el pasado. El lugar de una mujer en la sociedad debe ser el que ella quiera.

8. Es bueno que en hoy en día la juventud tenga más libertad para "hacer sus propias reglas" y protestar en contra de las cosas que no les gustan.

9. Algún día, el distanciamiento de la tradición terminará siendo un error fatal.

10. A largo plazo, hacer lo correcto y respetar la ley es mejor que estar desafiando constantemente las bases de nuestra sociedad.

11. Las personas deberían desarrollar sus propias normas sobre el bien y el mal, y poner menos atención a los textos antigüos que les enseñan lo que deben hacer.

12. Las relaciones homosexuales de largo plazo deberían tratarse igual que el matrimonio.

\section{ESCALA DE BÚSQUEDA EXISTENCIAL}

Existential Quest Scale (Van Pachterbeke, Keller y Saroglou, en prensa).

A continuación le presentamos una serie de afirmaciones que pueden o no aplicar en su caso. Por favor encierre en un círculo el número del 1 al 7 que mejor indica su grado de acuerdo o desacuerdo con cada afirmación.

1. Hoy en día, todavía me pregunto acerca del significado y la meta de mi vida.

2. Es probable que mi actitud hacia la religión/espiritualidad cambie de acuerdo con las experiencias de mi vida.

3. Ser capaz de dudar de nuestras propias convicciones y revalorarlas es una buena cualidad.

4. En mi opinión, cuando se trata de asuntos existenciales, dudar es importante.

5. Mi manera de ver el mundo definitivamente va a cambiar de nuevo.

6. Mi opinión acerca de muchos temas es variable.

7. Sé perfectamente cuál es la meta de mi vida.

8. Los años pasan, pero mi manera de ver el mundo no cambia.

9. A menudo revaloro mi opinión acerca de las creencias religiosas/espirituales. 


\section{ESCALA DE PRÁCTICAS RELIGIOSAS} cía, 2008).

Religiosity Scale (Saroglou y Muñoz-Gar-

A continuación le presentamos una serie de afirmaciones que pueden o no aplicar en su caso. Por favor encierre en un círculo el número del 1 al 7 que mejor indica su grado de acuerdo o desacuerdo con cada afirmación.

1. La religión es importante para mí.

2. Dios es importante para mí.

3. La espiritualidad es importante para mí.

Usted reza o hace oración: (Encierre en un círculo el número de la opción que elija)

\section{Nunca}

2. Casi nunca (por ejemplo, en momentos excepcionales de su vida)

3. Ocasionalmente (3-4 veces al año)

4. Varias veces al año

5. A menudo (varias veces al mes)

6. Muy a menudo (varias veces a la semana)

7. Todos los días

\section{ESCALA DE FUNDAMENTALISMO RELIGIOSO}

Religious Fundamentalism Scale (Altemeyer y Hunsberger, 2004).

Por favor indique el grado de acuerdo que usted tiene con las siguientes afirmaciones.

1. Dios ha dado a la humanidad una guía completa e infalible para la felicidad $y$ la salvación, que debe ser seguida totalmente.

2. Las enseñanzas religiosas acerca de las verdades intrínsecas y fundamentales de la vida, no están contenidas en un solo libro (R).

3. La causa básica del mal en este mundo es Satanás, que todavía está en lucha constante y feroz en contra de Dios.

4. Es más importante ser una buena persona, que creer en Dios y en la religión verdadera $(\mathrm{R})$.

5. Hay un conjunto particular de enseñanzas religiosas en este mundo que es muy cierto. No se puede ir "más profundo" porque es la base y el fundamento del mensaje que Dios ha dado a la humanidad.

6. Si uno lo piensa bien, hay básicamente dos tipos de personas en el mundo: los que hacen lo correcto, que serán recompensados por Dios, y el resto, que no obtendrá esta recompensa.

7. Para llevar la mejor vida y darle más sentido, uno debe pertenecer a la religión fundamentalmente verdadera.

8. "Satanás" es sólo el nombre que las personas dan a sus propios malos impulsos. Realmente no hay tal cosa como un diabólico "Príncipe de las Tinieblas", que nos tienta $(\mathrm{R})$.

9. Cuando hay conflictos entre la ciencia y los textos sagrados, probablemente la ciencia tenga la razón (R).

10. Los fundamentos de la religión de Dios nunca deben verse perjudicados por las creencias de los demás.

11. Todas las religiones del mundo tienen defectos $y$ enseñanzas equivocadas. No existe la religión perfectamente verdadera $y$ correcta $(\mathrm{R})$.

\section{ESCALA DE CUATRO POLOS DE LA RELIGIOSIDAD}

Four Poles Religiosity Scale (Saroglou, 2011).

Usted puede o no estar interesado/a en la religión por una variedad de razones. Por favor, trate de ser lo más específico/a posible en sus respuestas a las siguientes preguntas acerca de las razones que, eventualmente, le harían interesarse en la religión.

1. Una de las cosas que disfruto o disfrutaría de la religión es pertenecer a un grupo/comunidad.

2. Pertenecer a una tradición religiosa e identificarme con ella es o sería importante para mí.

3. Tener como referente una tradición religiosa es o sería importante para mi identidad étnica/cultural.

4. Soy o sería religioso/a debido a la ética y a los valores que la religión conlleva.

5. La religión me ayuda o me ayudaría a tratar de vivir orientado/a por valores morales. 
6. Cuando tengo un dilema moral, la religión me ayuda o me ayudaría a tomar una decisión.

7. Me gustan o me gustarían las ceremonias religiosas.

\section{ESCALA DE SATISFACCIÓN CON LA VIDA}

The Satisfaction with Life Scale (Diener, Emmons, Larsen y Griffin, 1985).

Abajo encontrará cinco afirmaciones con las que usted puede estar de acuerdo o en desacuerdo. Indique su grado de acuerdo con cada una.

1. En la mayoría de cosas, mi vida se acerca a mi ideal.

2. Las condiciones de mi vida son excelentes.

3. Estoy satisfecho/a con mi vida.

4. Hasta ahora he conseguido las cosas importantes que quiero en la vida.
5. Si pudiera vivir mi vida de nuevo, no cambiaría casi nada.

ESCALA DE ORIENTACIÓN E INTERESES POLÍTICOS

Political Interest and Orientation (Halman, 2001).

Por favor indique el grado de confianza que usted personalmente tiene en las siguientes instituciones.

1. La Asamblea Legislativa

2. La policía

3. Los políticos

4. Los partidos políticos

5. Las Naciones Unidas

En política, algunas veces, la gente habla de "izquierda" y "derecha”. ¿Dónde se colocaría usted en esta escala, en la cual 00 significa la izquierda y 10 significa la derecha? 
